\title{
On the Justification and Validity of the Kennard Inequality
}

\author{
Haengjin Choe \\ Department of Physics, College of Natural Sciences, Chonnam National University, Gwangju, South Korea
}

\section{Email address:}

eupjeong@gmail.com

\section{To cite this article:}

Haengjin Choe. On the Justification and Validity of the Kennard Inequality. American Journal of Modern Physics. Vol. 9, No. 5, 2020, pp. 73-76. doi: 10.11648/j.ajmp.20200905.12

Received: November 5, 2020; Accepted: November 23, 2020; Published: December 4, 2020

\begin{abstract}
In 1927, Earle Hesse Kennard derived an inequality describing Heisenberg's uncertainty principle. Since then, we have traditionally been using the standard deviation as the measure of uncertainty in quantum mechanics. But Jan Hilgevoord asserts that the standard deviation is neither a natural nor a generally adequate measure of quantum uncertainty. Specifically, he asserts that the standard deviations are inadequate to use as the quantum uncertainties in the single- and double-slit diffraction experiments. He even tells that from these examples it will become clear that the standard deviation is the wrong concept to express the uncertainty principle generally and that the Kennard relation has little to do with the uncertainty principle. We will investigate what are adequate as the measures of quantum uncertainty. And, beyond that, we will investigate the effects of multiplying the two uncertainties; namely, characteristics which is hiding in deep interior of the Kennard inequality. Through investigations we'll come to naturally realize that his assertions were wrong. All of our discussions will help raise understanding of the Heisenberg uncertainty principle. Our discussions will afford us an opportunity to think about the essence of the Fourier transform. The aim of this paper is to draw conclusions about whether the Kennard inequality is justified or not.
\end{abstract}

Keywords: Kennard Inequality, Quantum Uncertainty, Uncertainty Relation, Uncertainty Principle, Absolute Deviation, Standard Deviation

\section{Introduction}

Heisenberg's uncertainty principle [1] is one of the most famous foundations of quantum mechanics. Shortly after the publication by Werner Heisenberg, Earle Hesse Kennard derived $\sigma_{x} \sigma_{p} \geq \hbar / 2$ describing Heisenberg uncertainty principle, where $\sigma_{x}$ and $\sigma_{p}$ are the standard deviations of position $x$ and momentum $p$ [2]. Since then, we have traditionally been using the Kennard inequality as the uncertainty relation [3-11]. However Jos B. M. Uffink and Jan Hilgevoord assert that using the standard deviation and the Kennard inequality is not generally justified [12, 13]. Specifically, Hilgevoord asserts that using the standard deviation in the experiments in which electrons are incident on a slit is unjustified [13]. In regard to such a historical fact, we can doubt the followings: is there the reason why we use the standard deviation as the measure of uncertainty in quantum mechanics?; may we use some other quantities instead of using the standard deviation as the measure of quantum uncertainty?; if we may use some other quantities instead of the standard deviation, what are merits and demerits of each measure?; and so on. This article aims to arrive at rational conclusions on the justification and validity of the Kennard inequality by answering these questions. Finally, we will be able to better understand the essence of Heisenberg uncertainty principle.

\section{Discussions}

\subsection{Measures of Spread in Mathematical Statistics}

The author makes it clear that all of the contents stated in current section are facts on usual mathematical statistics which has absolutely nothing to do with quantum mechanics. Let us recall some results from mathematical statistics. When a statistical distribution is given, there are various measures of spread: standard deviation, absolute deviation, and so on [14]. Note that absolute deviation in this article indicates absolute mean deviation which is defined as the average of the absolute deviations taken from mean. The quantity such as the sixth root of the average of the sixth powers of deviations is also possible as a measure of spread, although this measure is complicated. In this 
way, we can define infinitely many measures of spread. In a purely notional aspect, we can tell that the absolute deviation of all possible measures of spread is the most ideal measure. But the absolute deviation is intricate to calculate; instead, we get around such a problem by adopting the standard deviation. Moreover, the standard deviation and various mathematical quantities build the simple relations such as the Chebyshev inequality [15]. Consequently, the advantage of being algebraically simpler is further highlighted than the advantage of being notionally more ideal. We eventually adopt the standard deviation as the measure of spread. Bear in mind that regardless of the shape of the curve of a statistical distribution, the defining equation of measure of spread is not changed. Additionally, when we determine what to adopt as the measure of spread, the shape of the curve of the distribution doesn't affect the criteria of our choice.

\subsection{Absolute Deviation as a Measure of Quantum Uncertainty}

Now let us return to quantum mechanics. We raised a series of questions in the introduction. In Section 2.1, we can get clues to the solutions of those questions. The standard deviation has traditionally been used as the measure of quantum uncertainty [3-11]. The standard deviation is merely one of the measures of spread. But, if we only purely notionally consider what to adopt as the measure of quantum uncertainty, the absolute deviation is more ideal than the standard deviation. But in quantum mechanics as in usual statistics, the absolute deviation is algebraically much more complex than the standard deviation. Let's take a look at the mathematical complexness of the absolute deviation through many concrete examples. Calculating the product of the two uncertainties of canonical variables $x$ and $p$ will be sufficient. Table 1 shows the products of the two uncertainties in two situations: the first situation of adopting the standard deviation and the second situation of adopting the absolute deviation. The second column indicates the products of the two standard deviations for various wavefunctions in the situation that we adopt the standard deviation. The third column indicates the products of the two absolute deviations for various wavefunctions in the situation that we adopt the absolute deviation. Because $k$ is a wavenumber, the Dirac constant $\hbar$ doesn't appear in Table 1.

Table 1. The products of the two uncertainties for various wavefunctions.

\begin{tabular}{|c|c|c|}
\hline wavefunction & $\begin{array}{l}\sqrt{\left\langle x^{2}\right\rangle-\langle x\rangle^{2}} \times \\
\sqrt{\left\langle k^{2}\right\rangle-\langle k\rangle^{2}}\end{array}$ & $\langle|x-\langle x\rangle|\rangle \times\langle|k-\langle k\rangle|\rangle$ \\
\hline$\frac{\sqrt{\frac{\pi}{a}}}{2 \cosh \frac{\pi x}{2 a}}$ & $\frac{\pi}{6}=0.524$ & $\frac{2\left(\log _{e} 2\right)^{2}}{\pi}=0.306$ \\
\hline$e^{-A x^{2}} \sqrt{\frac{2 A}{\pi}}$ & $\frac{1}{2}=0.5$ & $\frac{1}{\pi}=0.318$ \\
\hline$\frac{b}{b^{2}+x^{2}} \sqrt{\frac{2 b}{\pi}}$ & $\sqrt{\frac{1}{2}}=0.707$ & $\frac{1}{\pi}=0.318$ \\
\hline $\begin{array}{l}\sqrt{\frac{8}{45} x^{6} e^{-2 x}} \\
0 \leq x\end{array}$ & $\frac{\sqrt{7}}{2 \sqrt{5}}=0.592$ & $\left(\frac{7}{e}\right)^{7} \frac{1}{675 \pi}=0.354$ \\
\hline$\sqrt{\frac{4}{3} x^{4} e^{-2 x}}$ & $\frac{\sqrt{5}}{2 \sqrt{3}}=0.645$ & $\left(\frac{5}{e}\right)^{5} \frac{1}{18 \pi}=0.372$ \\
\hline
\end{tabular}

\begin{tabular}{|c|c|c|}
\hline wavefunction & $\begin{array}{l}\sqrt{\left\langle x^{2}\right\rangle-\langle x\rangle^{2}} \times \\
\sqrt{\left\langle k^{2}\right\rangle-\langle k\rangle^{2}}\end{array}$ & $\langle|x-\langle x\rangle|\rangle \times\langle|k-\langle k\rangle|\rangle$ \\
\hline \multicolumn{3}{|l|}{$0 \leq x$} \\
\hline $\begin{array}{l}\left(a^{2}-x^{2}\right) \sqrt{\frac{15}{16 a^{5}}} \\
|x| \leq a\end{array}$ & $\sqrt{\frac{5}{14}}=0.598$ & $\frac{75}{64 \pi}=0.373$ \\
\hline $\begin{array}{l}\left(x-x^{2}\right) \sqrt{30} \\
0 \leq x \leq 1\end{array}$ & $\sqrt{\frac{5}{14}}=0.598$ & $\frac{75}{64 \pi}=0.373$ \\
\hline $\begin{array}{l}\sqrt{4 x^{2} e^{-2 x}} \\
0 \leq x\end{array}$ & $\frac{\sqrt{3}}{2}=0.866$ & $\left(\frac{3}{e}\right)^{3} \frac{1}{\pi}=0.428$ \\
\hline
\end{tabular}

We can see from this table that when we adopt the absolute deviation as the measure of quantum uncertainty, the result values of multiplying the two uncertainties are relatively more intricate numbers. As we might expect, not only the result values but also intermediate calculation processes are more intricate. This one fact tells a great deal about justification of the standard deviation adoption. Thus we come to an important conclusion: from a perspective of algebraical complexity, the standard deviation is the best measure of quantum uncertainty. But algebraic simplicity isn't everything. In fact, there are much more important considerations which soon we will discuss. Even though algebraic simplicity is an important consideration, it is never the essential consideration. By the way, there is an interesting fact about the Gaussian wavefunction. You know, when we adopt the standard deviation as the measure of quantum uncertainty, the Gaussian wavefunction hits the position-momentum uncertainty limit. But, note that if we adopted the absolute deviation, it would be very difficult to know what is the minimum-uncertainty wavefunction.

\subsection{Standard Deviation as a Measure of Quantum Uncertainty}

We are now in a position to discuss the essence of the Kennard inequality in earnest. While Uffink and Hilgevoord assert that in the slit diffraction experiments, the standard deviation is not adequate as the measure of quantum uncertainty, they propose certain concepts related to the width of a function as an alternative [12, 13]. They try to express mathematically the uncertainty principle using those concepts [12]. Hilgevoord concludes that the Kennard inequality is not always an adequate expression of the uncertainty principle [13]. Hilgevoord seems to believe that we must be able to explain the uncertainty principle using the concept of the width of a function. However, he is losing sight of at least three facts. One fact which he is missing is that for us to explain the uncertainty principle by drawing the width of a function is in order to explain the principle roughly, not rigorously. Even if we confine our discussion to the square-integrable wavefunctions, our explanation by drawing is rough explanation. Of course! Mathematicians have not given a general definition to the width of a function as well. But, even though there is not the rigorous concept of the width of a function, or, even if we do not use at all the concept of the width of a function, we have no trouble not only describing the physical meaning of the uncertain principle but also describing mathematically the uncertain principle. Such a fact 
will become increasingly clear, as our discussion proceeds.

Another fact which Hilgevoord is missing is that the Fourier transform has an extraordinary ability to control the measure of spread. Obviously, he is underestimating such an ability of the Fourier transform. He asserts that because the standard deviation gives a large weight to the tails of a distribution, the standard deviation is not an adequate measure [13]. But the defining equation of a measure of spread is not affected by the shape of the curve of a statistical distribution. The logic of his arguments is extremely similar to the following argument: when we evaluate the area under the graph of a function over an interval, the formula to calculate the area is influenced by the shape of the graph. Of course, actually, the formula which calculates the area isn't influenced by the shape of the graph. All you have to do is to perform the definite integral without caring about the tails of the distribution curve. Don't care about the shape of the graph of the original function, and find the antiderivative of the function. Even if we accept to some extent his worry about the weight, it will result in no problem at all. The reason for no problem is due to the extraordinary ability of the Fourier transform. We perform the Fourier transform to obtain other variable which constitutes a pair of canonical variables [15]. At this point the Fourier transform removes his worry. A detailed discussion of this will be continued in the next paragraph.

The other fact which Hilgevoord is missing is about the effects and/or results of multiplying the two uncertainties. It seems that he has not thought deeply about the validity of multiplying the two uncertainties. We will be able to say that a superficial reason why we multiply the two uncertainties is the fact that the product of position and momentum has the same units as the product of energy and time. Moreover, we can additionally think over a more important reason which approaches the essence of the Kennard inequality. This essential reason is closely related with our discussion in the previous paragraph. View Table 1 again. We can see that the range of the results of multiplications for many wavefunctions is narrow, although there is a case, such as a particle in the infinite square well, in which the product of the two uncertainties infinitely increases with increasing quantum number. The result values of multiplication are in the range of about $0.3 \sim 0.9$ for the wavefunctions given in Table 1. Here we can realize the mighty power of the Fourier transform. Regardless of whether we adopt the standard deviation or the absolute deviation, and no matter which wavefunction we choose, the values of the product of the two uncertainties in the table are roughly equal; namely, there is a tendency for the value of the uncertainty of one variable to be inversely proportional to the value of the uncertainty of the other variable. This tendency originates in amazing ability of the Fourier transform to control the spread of data. Therefore, we don't have to think seriously of the shape of the curve of a distribution. This is the very mighty power of the Fourier transform.

\subsection{Confirmation of the Ideality of Absolute Deviation}

Because of the two facts, we can once again confirm that the absolute deviation is notionally more ideal than the standard deviation. The most important fact of the two facts is that for the same wavefunction, the value of multiplication in the situation of the absolute deviation is always smaller than the value of multiplication in the situation of the standard deviation. The other fact of the two facts is that the range of the results of the products in the situation of the absolute deviation is narrower than the range of the results of the products in the situation of the standard deviation: for the wavefunctions given in Table 1, the range is approximately $0.428-0.306=0.122$ in the situation of the absolute deviation and approximately $0.866-0.5=0.366$ in the situation of the standard deviation.

\subsection{Infimums in Uncertainty Relations}

For a long time, the Kennard inequality has been a strong expressive means to describe mathematically the uncertainty principle. By the way, it is always highlighted only that we can calculate the minimum value of the products of the two uncertainties. However, the author thinks that for the range of the result values of the products to be narrow is more important than for us to be able to calculate the minimum value of the result values. If we adopt the absolute deviation, it seems that it is almost impossible to obtain the infimum of the products of the two uncertainties. Of course, even if so, the interpretation and physical meaning of the uncertainty principle are maintained.

\section{Conclusions}

As we have seen in Section 2.1, the shape of the curve of a distribution in usual statistics doesn't affect the defining equation of a measure of spread. The same goes for quantum mechanics. It is not reasonable that we are into the concept of the width of a function so we consider measures of quantum uncertainty according to the shape of the curve of a distribution. Another reason why we needn't consider the width of a function is based on the nature of the Fourier transform. In conclusion, it is fully reasonable that we adopt the standard deviation as the measure of spread putting a lot of value on simplification of mathematical treatment. Although notionally less ideal than the absolute deviation, the standard deviation is algebraically more simple and hence is more practical. The adoption of the standard deviation greatly simplifies the calculation of the product of the two uncertainties. Again, we can use the existing measures of spread in order to express mathematically the uncertainty principle.

Since the publication of Kennard, we have traditionally been using the standard deviation as the measure of uncertainty in quantum mechanics. Fourier transform has its own ability to control the spread of data. The products of the measures of spread of the two canonical variables are roughly equal. This nature of the Fourier transform justifies constructing the uncertainty relation by multiplying the two 
uncertainties. Specifically, the nature of the Fourier transform justifies the Kennard inequality. We may adopt other quantities instead of the standard deviation as measure of quantum uncertainty. For example, we can adopt the absolute deviation. However, if we adopt the absolute deviation, the infimum of the product of the absolute deviations of two variables would be next to impossible to obtain. But it is not a serious problem. Even if we can't obtain the infimum, the uncertainty principle doesn't crack. Consequently, we will not need to put too many overtones on the constant value, $\hbar / 2$, given as the minimum value. The most important fact in the uncertainty relation is that, regardless of the measure of uncertainty, neither of the two uncertainties multiplied are 0 . This fact is the very essence of the uncertainty principle.

\section{References}

[1] W. Heisenberg, "Über den anschaulichen Inhalt der quantentheoretischen Kinematik und Mechanik," Z. Physik, vol. 43, pp. 172-198, March 1927.

[2] E. H. Kennard, "Zur Quantenmechanik einfacher Bewegungstypen,” Z. Physik, vol. 44, pp. 326-352, April 1927.

[3] D. J. Griffiths and D. F. Schroeter, Introduction to Quantum Mechanics, 3rd ed., Cambridge University Press, 2020.

[4] E. Merzbacher, Quantum Mechanics, 3rd ed., Wiley, 1998.
[5] J. J. Sakurai, Modern Quantum Mechanics, Rev. ed., Addison-Wesley, 1994.

[6] M. A. de Gosson, The Principles of Newtonian and Quantum Mechanics, 2nd ed., World Scientific, 2017.

[7] A. Kumar, Fundamentals of Quantum Mechanics, Cambridge University Press, 2018.

[8] J. Baggott, The Quantum Cookbook, Oxford University Press, 1st ed., 2020.

[9] P. L. Bowers, Lectures on Quantum Mechanics, Cambridge University Press, 2020.

[10] S. J. Gustafson and I. M. Sigal, Mathematical Concepts of Quantum Mechanics, 3 ed., Springer, 2020.

[11] J. Izaac and J. Wang, Computational Quantum Mechanics, Springer, 2018.

[12] J. B. M. Uffink, J. Hilgevoord, "Uncertainty principle and uncertainty relations," J. Found. Phys, vol. 15, pp. 925-944, September 1985.

[13] J. Hilgevoord, "The standard deviation is not an adequate measure of quantum uncertainty," Am. J. Phys, vol. 70, pp. 983 , October 2002.

[14] B. L. Agarwal, Basic Statistics, 4th ed., New Age International Publishers, 2006, pp. 61-81.

[15] G. B. Arfken, H. J. Weber, and F. E. Harris, Mathematical Methods for Physicists, 7th ed., Elsevier, 2013. 